\title{
MEMORANDUM
}

RM-5193-NASA

APRIL 1967

\section{COMPARISON OF ESTIMATES OF SURFACE FLUID MOTIONS OF THE EARTH'S CORE FOR VARIOUS EPOCHS}

Anne B. Kahle, R. H. Ball and E. H. Vestine

This research is sponsored by the National Aeronautics and Space Administration under Contract No. NASr-2l. This report does not necessarily represent the views of the National Aeronautics and Space Administration. 


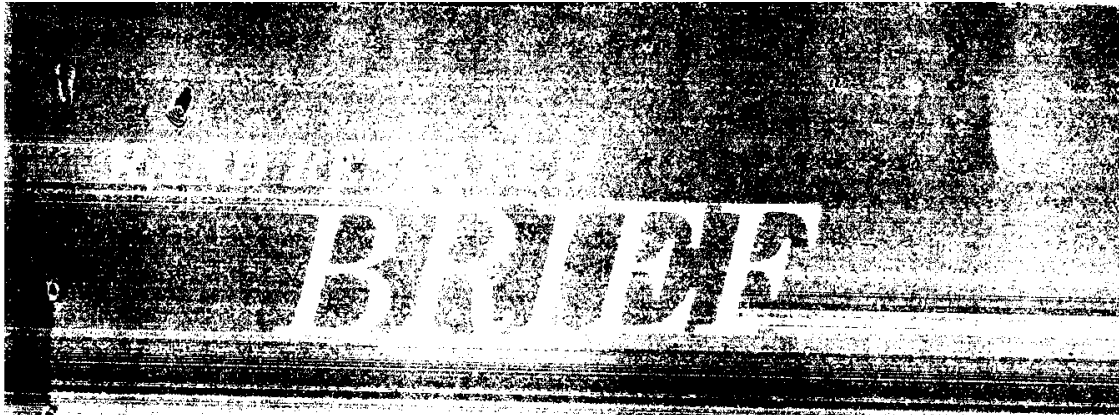

Apri1 1967

$\mathrm{RB}-5193$

RM-5193-NASA, Comparison of Estimates of Surface Fluid Motions of the Earth's Core for Various Epochs, Anne B. Kahle, R. H. Ball, and E. H. Vestine, RAND Memorandum, Apri1 1967, 25 pp.

PURPOSE: To estimate and compare the velocity of the fluid at the surface of the earth's core for different epochs since 1885 .

RELATED TO: RAND's research for the National Aeronautics and Space Administration. This study is one of a series seeking to improve predictions of the geomagnetic field that dominates the radiation belts of the earth. The series is planned to assist in estimating magnetic fields likely to be encountered on other planets. Previously published studies include RM-5191-NASA, Westward Drift of the Geomagnetic Field and Its Relation to Motions of the Earth's Core, December 1966, and RM-5091-NASA, Estimated Surface Motions of the Earth's Core, August 1966.

METHODOLOGY AND DISCUSSION: The velocity of the fluid at the surface of the earth's core can be determined from the earth's main magnetic field and the secular, or longterm, change of the magnetic field with time. These changes are assumed to be due to the motion of the fluid, with the magnetic field "frozen" into it. The velocity field can be found by numerically solving the appropriate magnetohydrodynamic equation. This solution provides a useful approximation, but it neglects the generation of secular change caused by radial fluid flow reacting with toroidal fields near the core surface.

In previous studies, the secular-change field was averaged over the 43-year period from 1912 to 1955. The method is modified here, and the velocity field is determined and compared for several epochs since 1885. The instantaneous secularchange field corresponding to the appropriate epoch of the main field is used to determine the velocity in greater detail. The results are of interest in that they show how the velocity field changes with time and indicate the reliability of the velocities derived from the rather inaccurate secular-change fields.

PRINCIPAL FINDINGS: Comparison of the velocity patterns at epochs 1885, 1912, 1933, and 1960 shows the same basic feature-an upflow over most of the Southern Hemisphere. Probably the most striking feature is the strong, presistent upflow near the southern end of Africa. A second, smaller center of upflow occurs in the Southeast Pacific. The main center of the downflow is found to vary with epoch from the Central Pacific to South America to Europe and back to the Central Pacific again. The similarity of the 1885 velocity pattern to that of 1960 hints at either a repetition of the pattern or a real intermediate change near 1900. A pattern of four rotational cells, corresponding roughly to geostrophic flow around the vertical flow, seems to persist. Again there appears to be a strong similarity between 1885 and 1960 .

All epochs show a fairly strong westward velocity in the Southern Hemisphere with either a negligible or small eastward velocity in midlatitudes of the Northern Hemisphere. These patterns differ considerably from the generally accepted westward drift of about $0.2^{\circ}$ a year in all latitudes, averaging less than half that value. 


\section{PREFACE}

The fluid central portion of the earth contains the generator of the earth's main magnetic field. As the surface of the core moves about, it carries with it the magnetic field lines extending to and observable at the surface of the earth. We use the motions of the field lines to infer the surface motions in the core, which are estimated and compared for a number of years beginning in 1885 .

The present study is one of a series seeking to improve predictions of the geomagnetic field, which dominates the radiation belts of the earth, and to usefully assist in estimating magnetic fields likely to be encountered on other planets.

This work was supported by the National Aeronautics and Space Administration. 


\begin{abstract}
The velocity of the fluid at the surface of the earth's core can be related to the magnetic field and its secular change at the earth's surface through hydromagnetic equations. The method we developed previously for finding the velocity is briefly reviewed, along with a few modifications -- principally the use of the secular change for a single epoch rather than for a 40-year average. The velocity patterns for epoch 1960 show an upflow in southern Africa and a downflow in the Pacific, and four horizontal rotational cells, corresponding roughly to geostrophic flow around the vertical flow. The westward drift is somewhat smaller than that found previously by others. Comparison of the velocity patterns at epochs 1885, 1912, 1933, and 1960 shows the African upflow to be a strikingly persistent feature.
\end{abstract}




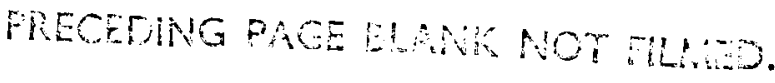 \\ -vii-}

\section{CONTENTS}

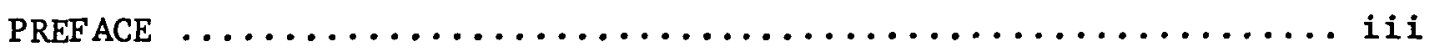

ABSTRACT $\ldots \ldots \ldots \ldots \ldots \ldots \ldots \ldots \ldots \ldots \ldots \ldots \ldots \ldots \ldots \ldots \ldots \ldots$

Section

I. INTRODUCTION $\ldots \ldots \ldots \ldots \ldots \ldots \ldots \ldots \ldots \ldots \ldots \ldots \ldots \ldots$

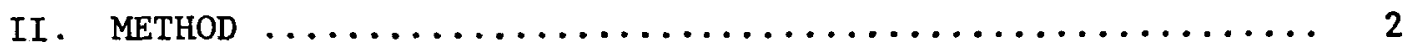

III. VARIATION OF VELOCITY WITH EPOCH $\ldots \ldots \ldots \ldots \ldots \ldots \ldots \ldots \ldots 11$

REFERENCES $\ldots \ldots \ldots \ldots \ldots \ldots \ldots \ldots \ldots \ldots \ldots \ldots \ldots \ldots \ldots \ldots \ldots \ldots \ldots \ldots$ 


\section{INTRODUCTION}

In two previous papers (Vestine and Kahle, 1966; Kahle, et a1., 1967) we have developed a method for determining the velocity of the fluid at the surface of the earth's core. The basic theory of the fluid velocity at the core boundary is similar to that of Roberts and Scott (1965). This velocity is found from the earth's main magnetic field and the secular, or long-term, change of the magnetic field with time. These changes are assumed to be due to the motion of the fluid, with the magnetic field "frozen" into it (Alfvén and Fälthammer, 1963). The velocity field can be found by numerically solving the appropriate magnetohydrodynamic equation. This solution is apt to provide a useful approximation, so long as we can neglect the generation of secular change caused by radial fluid flow reacting with toroidal fields near the core surface.

The method is modified somewhat here, and the velocity field is determined and compared for several epochs since 1885. In our previous work the secular change field was averaged over the 43-year period from 1912 to 1955. Now, in the hope of determining the velocity in greater detail, we use the instantaneous secular change field corresponding to the appropriate epoch of the main field. This is of interest both to show how the velocity field changes with time, and to give some indication of the reliability of the velocities derived from the rather inaccurate secular change fields. 


\section{METHOD}

The method of finding the velocity of the fluid at the core surface is briefly outlined here. A more detailed discussion can be found in a previous paper (Kahle, et al., 1967). A basic equation of magnetohydrodynamics (Cowling, 1956) is

$$
\frac{\partial \underset{B}{\sim}}{\partial t}=\nabla \times(\underset{\sim}{(v)} \underset{\sim}{B})+\frac{1}{4 \pi \sigma} \nabla^{2} \underset{\sim}{B}
$$

In the core, the first term on the right-hand side, the tranport term, apparently dominates over the second, diffusion, term. Hence we have the approximation

$$
\frac{\partial B}{\partial t}=\nabla \times(\underset{\sim}{\text { v }} \times \underset{\sim}{B})
$$

At the surface of the core the radial component of the velocity, $v_{r}$, must vanish, since we assume that there is no flow across the core-mantle interface. Making the further assumption that the fluid is incompressible $(\nabla \cdot \underset{\sim}{v}=0)$, and remembering that $\nabla \cdot \underline{\sim}=0$, we can express the radial component of $\mathrm{Eq}$. (2) in spherical coordinates as

$$
\begin{aligned}
-\frac{\partial \mathrm{B}_{r}}{\partial t} & =\frac{{ }^{B}}{r}\left(v_{\theta} \cot \theta+\frac{\partial v_{\theta}}{\partial \theta}+\frac{1}{\sin \theta} \frac{\partial v_{\lambda}}{\partial \lambda}\right) \\
& +\frac{v_{\theta}}{r} \frac{\partial{ }_{r}}{\partial \theta}+\frac{v_{\lambda}}{r \sin \theta} \frac{\partial{ }_{r}}{\partial \lambda}
\end{aligned}
$$

(Roberts and Scott, 1965; Kahle, et a1., 1967).

As before, we express the 2-dimensional (horizonta1) velocity in the general form

$$
\underset{\sim}{\mathrm{v}}=-\nabla_{\mathrm{T}} \psi+\underset{\sim}{\mathrm{r}} \times \nabla X
$$

where $\nabla_{\mathrm{T}}$ represents the 2 -dimensional gradient, $\psi$ is a potential function, and $X$ is a stream function. We express both $\psi$ and $X$ in spherical 
harmonic series :

$$
\begin{aligned}
& \psi=\underset{n}{ } \sum_{\mathrm{m}} \sum_{\mathrm{n}}\left(\alpha_{\mathrm{m}}^{\mathrm{m}} \cos \mathrm{m} \lambda+\beta_{\mathrm{n}}^{\mathrm{m}} \sin \mathrm{m} \lambda\right) \mathrm{P}_{\mathrm{n}}^{\mathrm{m}}(\theta) \\
& x=\sum_{\mathrm{n} m} \sum_{\mathrm{m}}\left(\mathrm{A}_{\mathrm{n}}^{\mathrm{m}} \cos \mathrm{m} \lambda+B_{\mathrm{n}}^{\mathrm{m}} \sin \mathrm{m} \lambda\right) \mathrm{P}_{\mathrm{n}}^{\mathrm{m}}(\theta)
\end{aligned}
$$

The velocity is found by solving Eq. (3) numerically for the fluid velocity coefficients $\alpha_{n}^{m}, \beta_{n}^{m}, A_{n}^{m}$, and $B_{n}^{m}$, accomplished by a least-squares fit of the data over the surface of the core. The data consist of the $\underset{\sim}{B}$ and $\underset{\sim}{\dot{B}}$ fields measured at the surface of the earth, expressed in spherical harmonic series, and extrapolated down to the core.

The core surface velocity derived in this manner for 1960 is shown in Figs. 1 through 4. The magnetic field data used are those for the main field with terms to $n=6$ for epoch 1960 and for the secular change field with terms to $\mathrm{n}=4$ for the same epoch, as given by Hendricks and Cain (1966).

Figure 1 shows that part of the velocity represented by the potential function $\psi$. The arrows show the horizontal vector velocity. The contours of equal $\psi$ can also be seen to indicate this velocity, which is always perpendicular to the contours, and stronger where the contours are closer. Since the fluid is assumed to be incompressible, this potential function also gives us some information about the vertical velocity. The sources (positive, solid contours) and sinks (negative, dotted contours) can be thought of as regions of upflow and downflow, respective $1 y$.

The rotational velocity, represented by the stream function $x$, can be further divided into two parts: an east--west velocity (rotation about the poles), which is given by the $A_{n}^{0}$ terms of Eq. (5), and the remainder of the rotational velocity, following paths closed on the surface of the core. Figure 2 shows this latter part of the velocity, both by vector arrows and by contours, or streamlines, of $x-A_{n}^{0}$. Note that this stream function velocity lies along the contours of $\chi^{n}-A_{n}^{0}$, rather than perpendicular to the contours as does the potential $\psi$. Again, the density of contour lines indicates the strength of the velocity. The flow is clockwise for positive $\chi$ (solid lines) and counterclockwise for negative $x$ (dotted lines). 


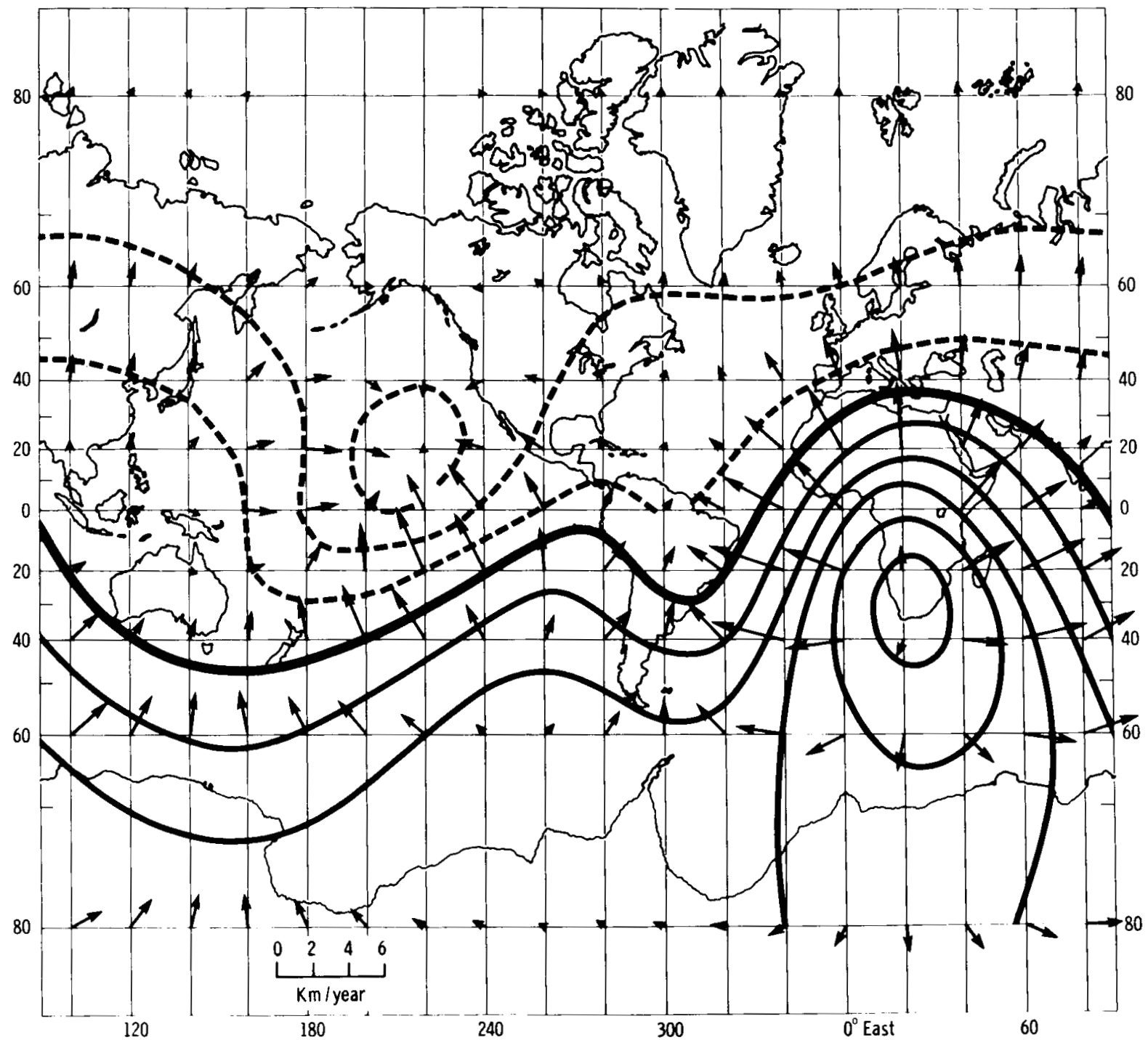

Fig. 1 - Irrotational part of core surface velocity for epoch 1960. 


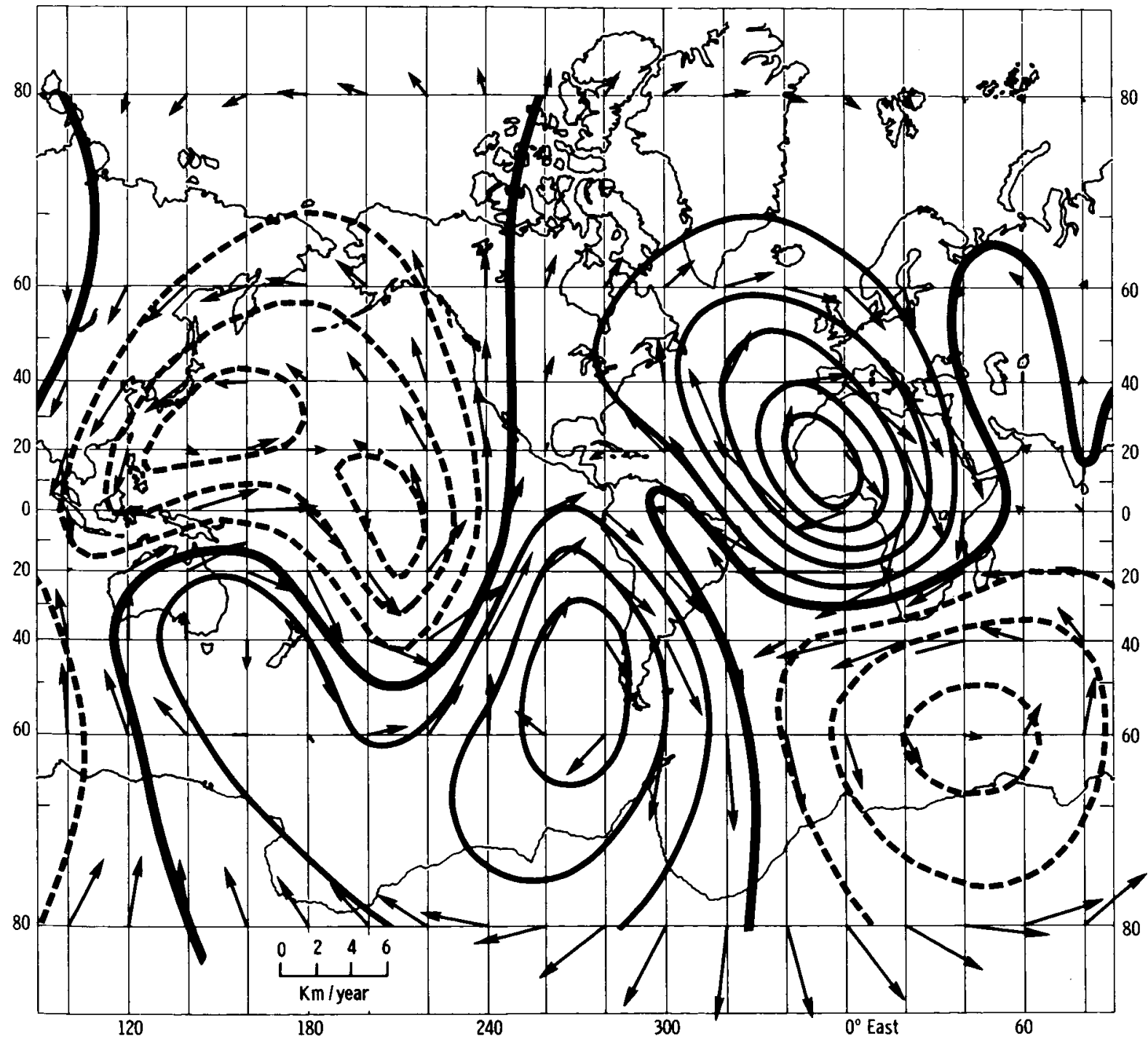

Fig. 2 -- Rotational part of core surface velocity minus the westward drift, for epoch 1960. 


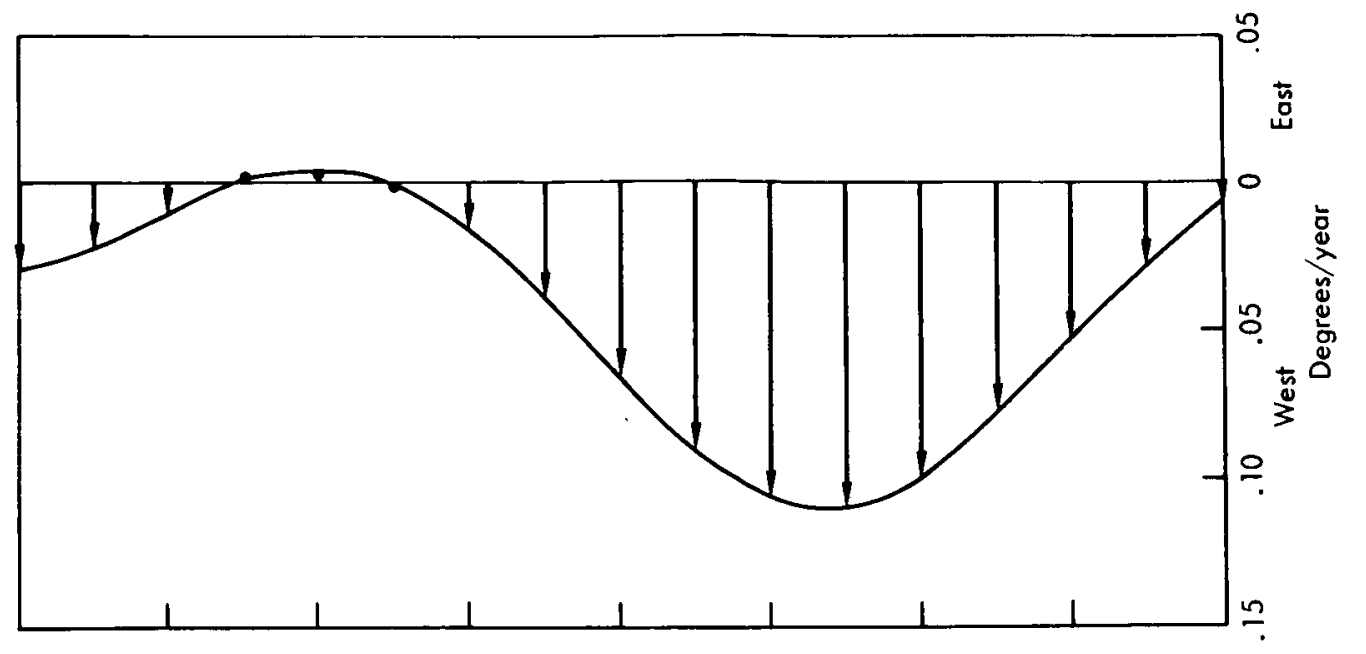

ᄋ̊

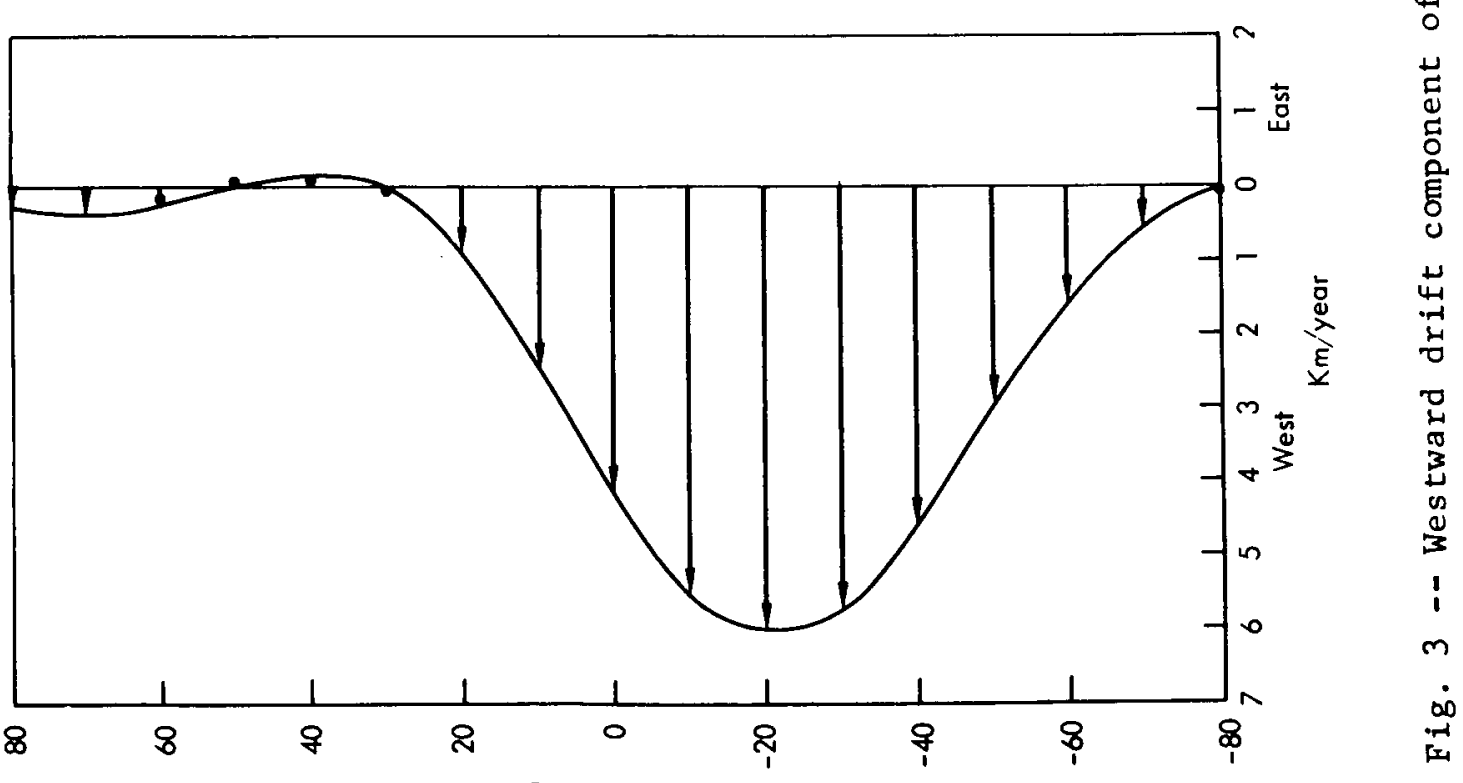

(6әp) әpก+!+0า 


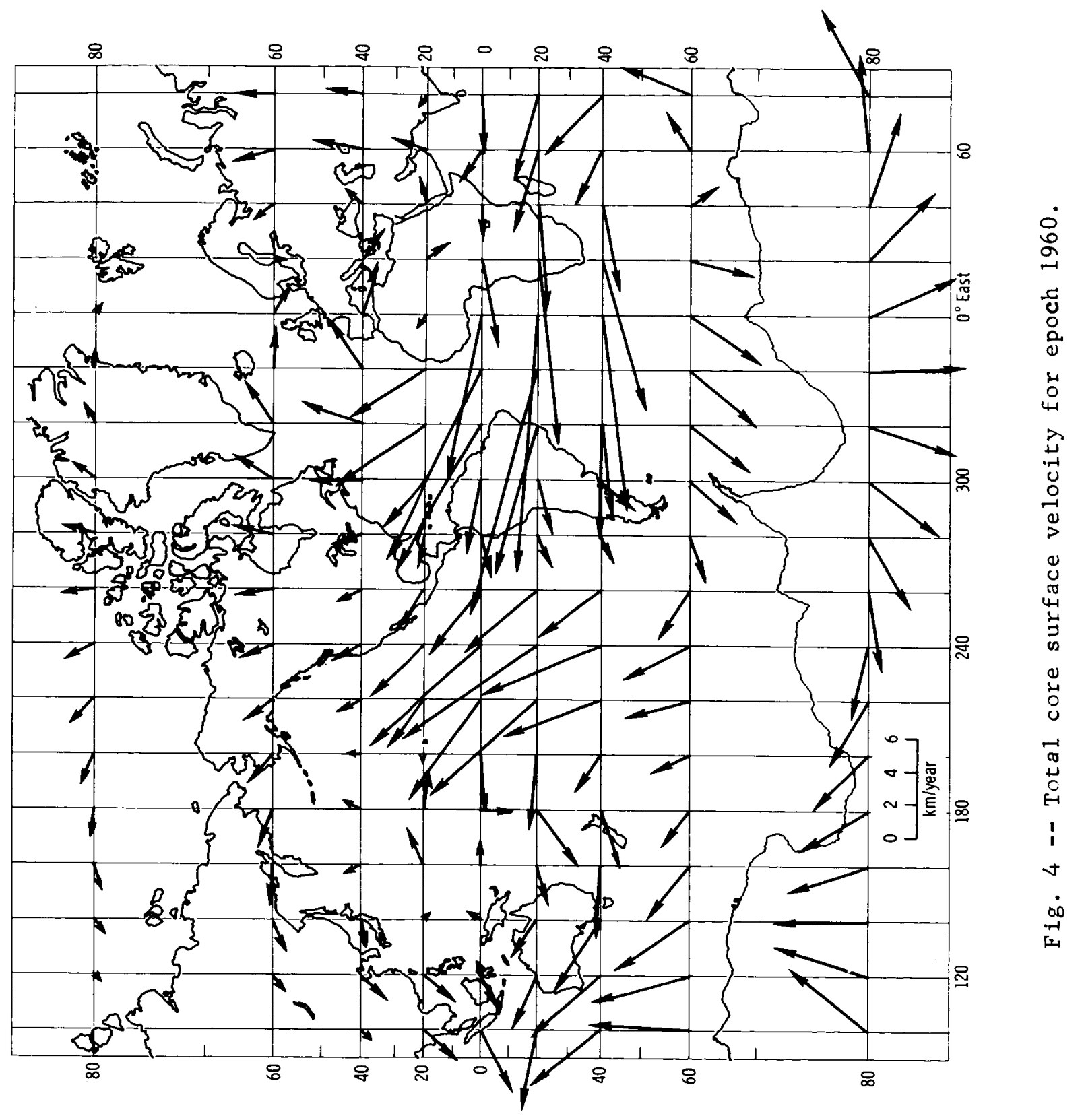


Figure 3 shows the east--west velocity as a function of latitude. This should correspond to the well-known westward drift. Although this drift is mostly westward, it is neither constant (in degrees/yr) with latitude, as in most previous determinations, nor is it as large as that found by others. Figure 4 shows the total velocity at the core surface for epoch 1960, which is the sum of the three velocities given in Figs. 1 through 3.

In our previous exploratory work, we did not extrapolate the magnetic field and the secular change field all the way down to the surface of the core at $r=3471 \mathrm{~km}$. Instead, we examined the velocity patterns at a hypothetical core surface at $r=4000 \mathrm{~km}$, assuming that the general patterns at that depth would be quite similar to those at the true core. In this way we hoped to reduce the amplification of error in the higher-degree harmonics of the extrapolated magnetic fields. Further study has shown, however, that extrapolation all the way to the core apparently does not change the values found for the coefficients of $\psi$ and $X$ very much. Therefore, in our present study we do examine the velocity patterns at the surface of the core $(r=3471 \mathrm{~km})$.

It is quite possible that not all the observed secular change is due to the magnetic field moving with the fluid. The change reflected in the observed long-term decay of the main dipole may be unrelated to fluid motions. Therefore, we tried removing the average of this decay from the dipole secular change field, leaving the residual $\dot{\mathrm{g}}_{1}^{0}, \dot{\mathrm{g}}_{1}^{1}$, and $\dot{h}_{1}^{1}$ terms, plus all the higher-order terms. The resulting velocity, however, was essentially unaffected, probably because these low-order terms become relatively unimportant when extrapolated to the core.

It should perhaps be mentioned that the whole main magnetic field and the whole secular change field are used in these calculations. This is, of course, because the moving fluid interacts with the whole magnetic field. This method differs from some other methods of finding velocities, which involve the relationship between only the nondipole part of the magnetic field and the secular change field (Bullard, et al., 1950; Yukatake, 1962; Nagata, 1962). A1though Bullard's work does give a reliable result for the westward drift of the nondipole part of the magnetic field, it is only a first approximation to the actual motion of 
the fluid at the surface of the core. Our method represents a higher approximation to this fluid flow. For comparison we used a simplified version of our method, in which the additional approximations of Bullard are used to determine a pure westward drift. Using only the nondipole main magnetic field and the nondipole secular change field, we solved Eq. (3) at the surface of the earth, rather than at the surface of the core. We restricted the velocity to a westward drift component by forcing the data to be fitted with $A_{n}^{0}$ terms only. The resulting westward drift, as shown in Fig. 5, is comparable to that found by Bullard, averaging just less than $0.2^{\circ} / \mathrm{yr}$.

The tangential flow at the surface of the core, estimated by our method using Eq. (3), is the flow that will generate the observed secular change at the earth's surface. Our results pertain only to a core for which the surface flow is fairly simple. A fine-grained surface flow configuration would not satisfy a frozen-field concept because the magnetic Reynolds number would be small. Even if such small-scale flow patterns do exist, their effect in $\mathrm{dB}_{\mathrm{r}} / \mathrm{dt}$ at the earth's surface would be almost negligible because of the extreme attenuation of signals by the electromagnetically conducting mantle. It should also be noted that we have not estimated the flow of fluid (if any) parallel to the isomagnetic lines of $B_{r}$ at the core's surface, since this flow fails to contribute to secular change. 


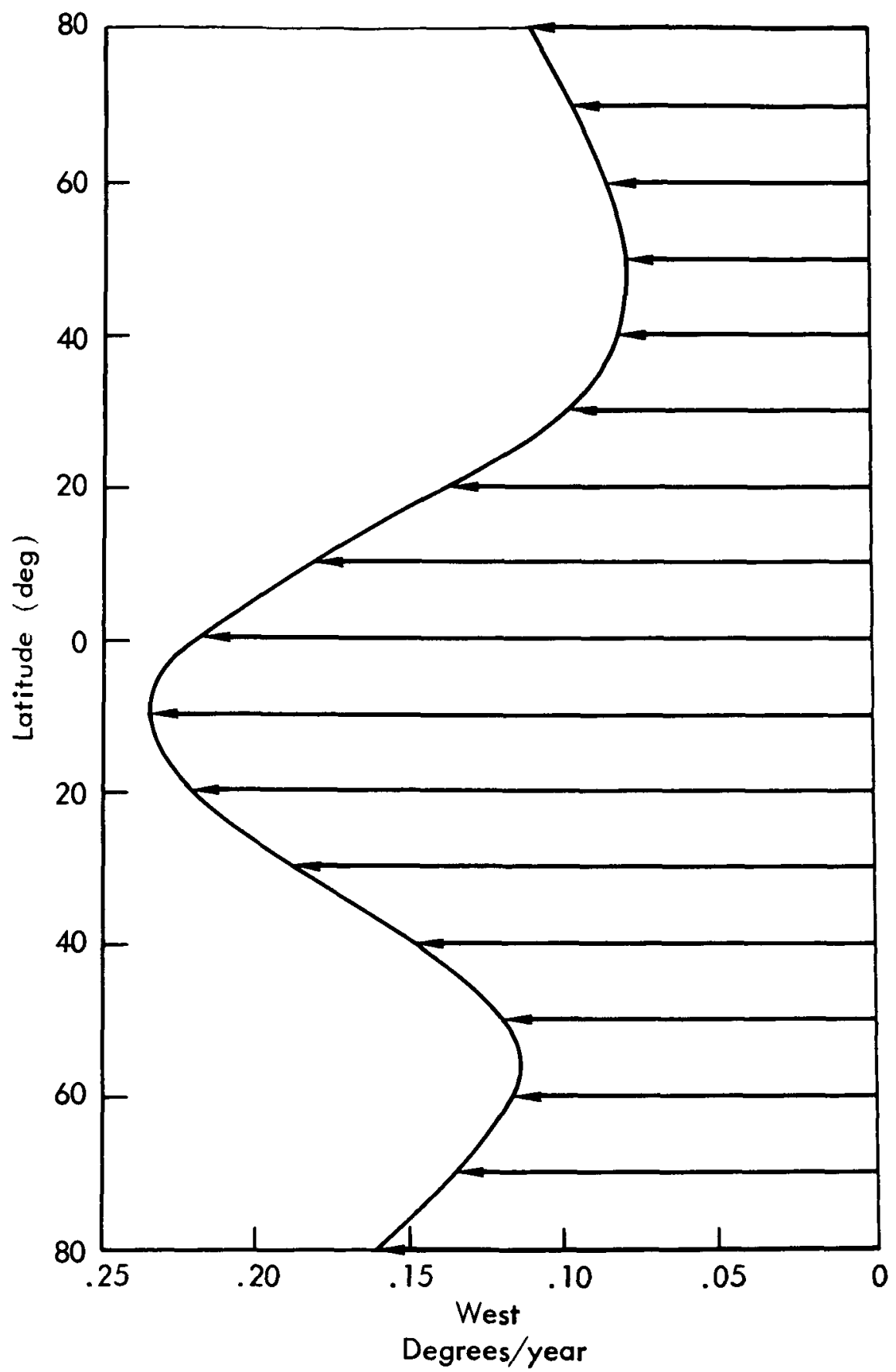

Fig. 5 -- Westward drift at earth's surface, using only nondipole fields and assuming only zonal flow. 


\section{VARIATION OF VELOCITY WITH EPOCH}

One aim of this study is to compare the velocity patterns at different epochs. For each epoch we have found the velocity at the core surface $(r=3471 \mathrm{~km})$, using the entire main magnetic field with terms up to $\mathrm{n}=6$, and the entire secular change field with terms up to $\mathrm{n}=4$. The epochs considered and the sources of the magnetic field data for the epochs are listed in Table 1.

Table 1

SOURCES OF DATA FOR VARIOUS EPOCHS

\begin{tabular}{ll}
\hline Epoch & \multicolumn{1}{c}{ Source of Data } \\
\hline 1885 & Mauersberger (1952) \\
1912 & Mauersberger (1952) \\
1933 & Vestine, et a1. (1947) \\
1960 & Hendricks and Cain (1966) \\
\hline
\end{tabular}

Figure 6 shows contours of the velocity potential $\psi$ for all epochs; this part of the velocity is perpendicular to the contours. All the patterns show the same basic feature -- a source or upflow over most of the Southern Hemisphere. Probably the most striking feature is the strong, persistent upflow near the southern end of Africa. The strength and position seem to vary only slightly, though it appears that there may be a westward motion of the center. There is also a second, smaller center of upflow in the southeast Pacific, but this shows considerably more variation. The centers of downflow are more poorly defined. The calculations suggest that they appear to be rather variable, but this finding may be misleading, and perhaps arises partly from magnetic field charting errors. The main center of downflow calculated here is found to vary with epoch from the central Pacific to South America to Europe and back to the central Pacific again. The similarity of the 1885 velocity pattern to that of 1960 hints at either a repetition of the pattern or a real intermediate change near 1900 . We hope to attempt later an 

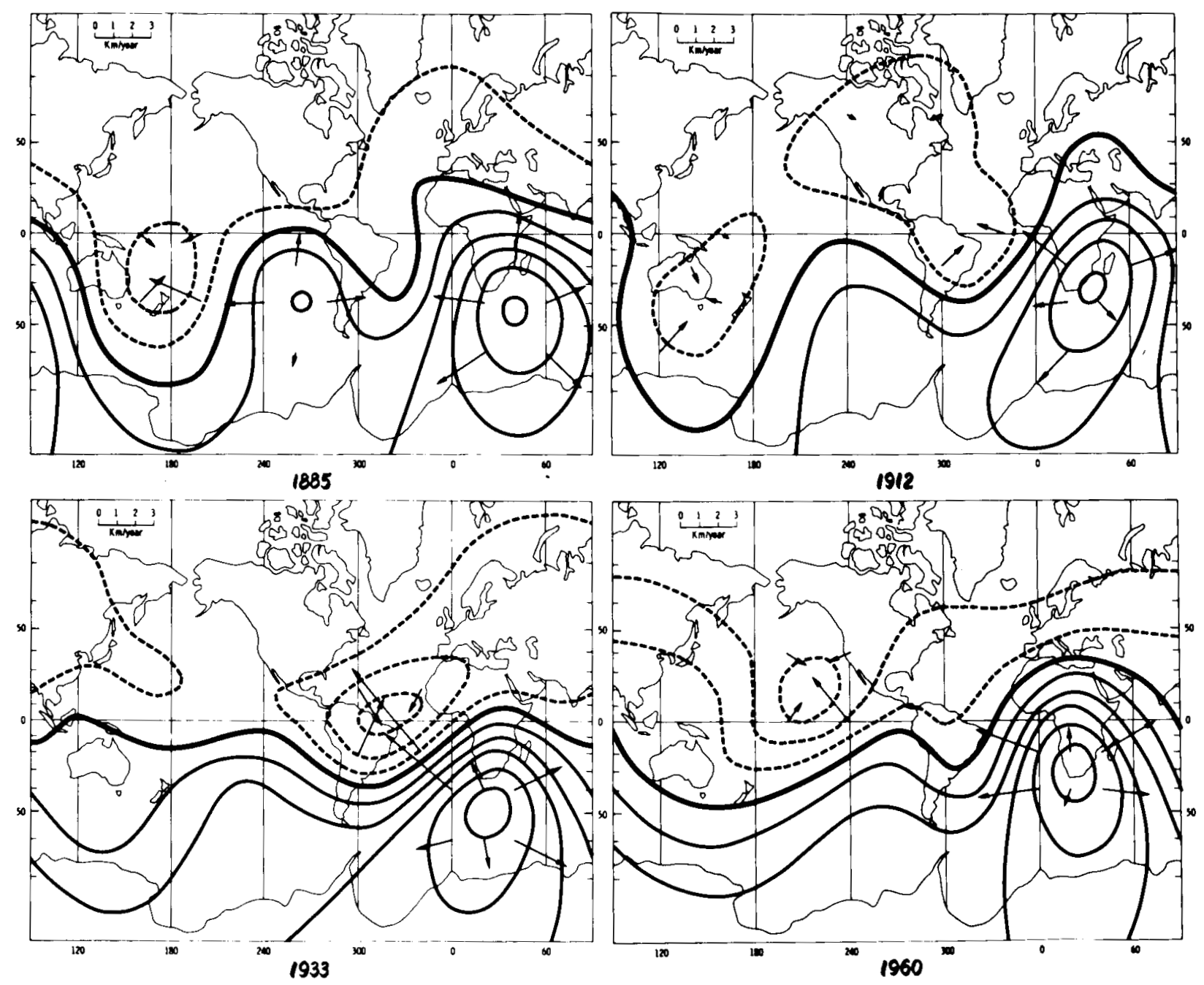

Fig. 6 -- Irrotational part of core surface velocity for epochs 1885, 1912,1933 , and 1960. 
examination of the latter possibility. Changes of pattern with time may also be related to the magnetohydrodynamic waves of Hide (1966). However, while we have reasonable confidence in the features that persist from one epoch to the next, we believe that the transient features should be regarded with some doubt, since they must -- at least in some degree -- reflect the presence of substantial systematic and other errors known to exist in magnetic charts.

Figure 7 shows the stream lines of $\chi$, where the velocity is now parallel to the lines. The contour intervals are twice as large as those shown in Fig. 6, since these rotational velocities are somewhat larger than the potential velocities of Fig. 6. The pattern that appears to persist here consists of four rotational cells: a counterclockwise cel1 south of Africa extending into the south Atlantic and South America; another in the central Pacific; a clockwise cell in Africa; and another in the south Pacific. The middle-epoch charts also show two additional cells north of these. Again there appears to be a strong similarity between 1885 and 1960. If one regards the rotational patterns as geostrophic in origin, due to Coriolis forces in regions of upflow and downflow, then the four main cells would correspond to an upflow in Africa and a downflow in the Pacific. This corresponds somewhat to the velocities in Fig. 6, although in general there is an additional northward component in the potential flow -- that is, the African source is in the Southern Hemisphere. Of course, all these patterns are only roughly defined, with considerable deviation from one epoch to the next.

The third part of the velocity, the east--west component, is shown in Fig. 8. All epochs show a fairly strong westward velocity in the Southern Hemisphere with either a negligible or small eastward velocity in mid-latitudes of the Northern Hemisphere. The large velocities in high latitudes, especially noticeable in the middle epochs, are not felt to be too reliable, due to the lack of good data in those regions, so we will confine our remarks to the mid-and low-latitude velocities. As noted in the previous section, these patterns differ considerably from the generally accepted westward drift of about $0.2^{\circ} / \mathrm{yr}$ in all latitudes. The westward velocities that we find average less than half that value. 

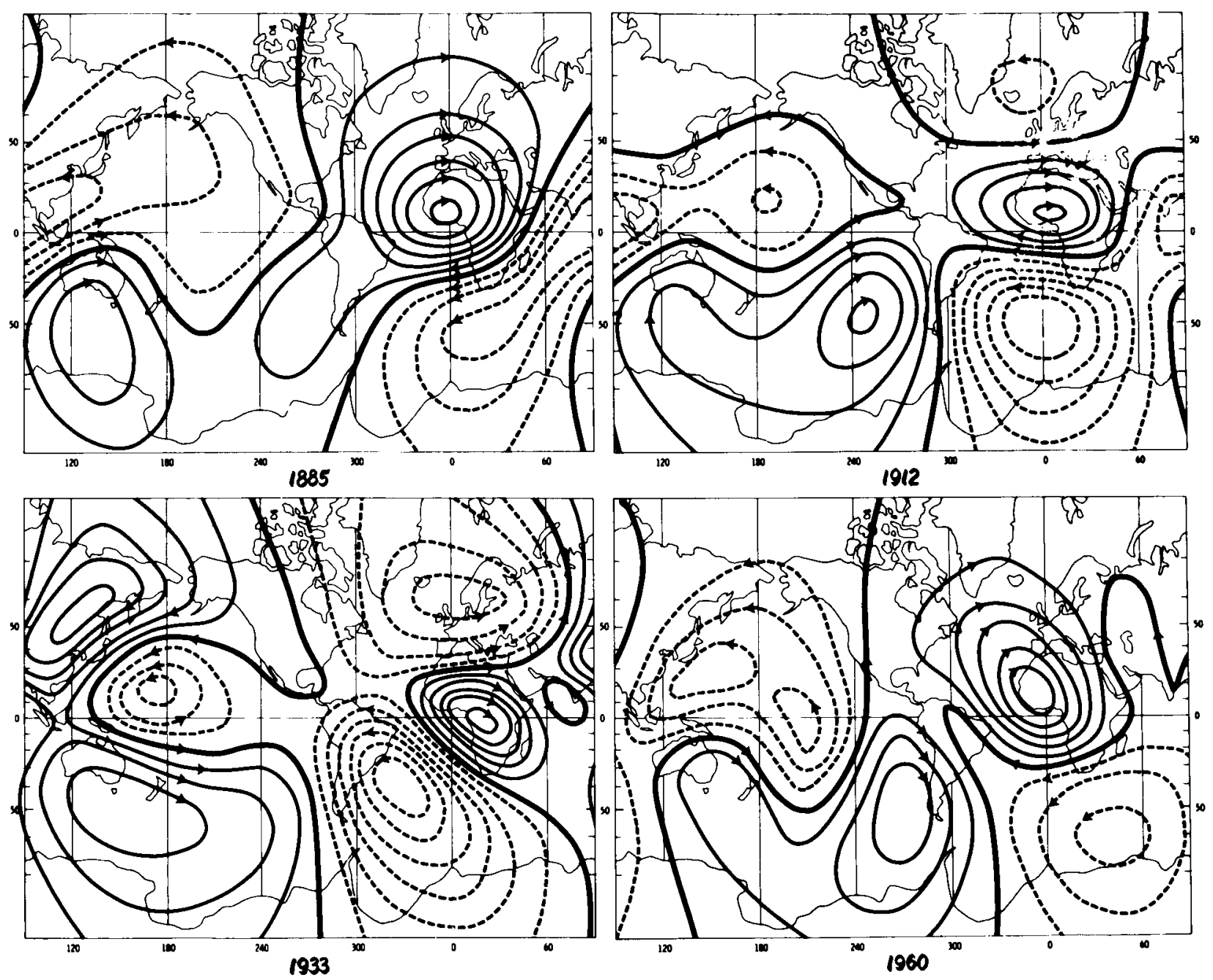

Fig. 7 -- Rotational part of core surface velocity minus the westward drift, for epochs 1885, 1912, 1933, and 1960 . 

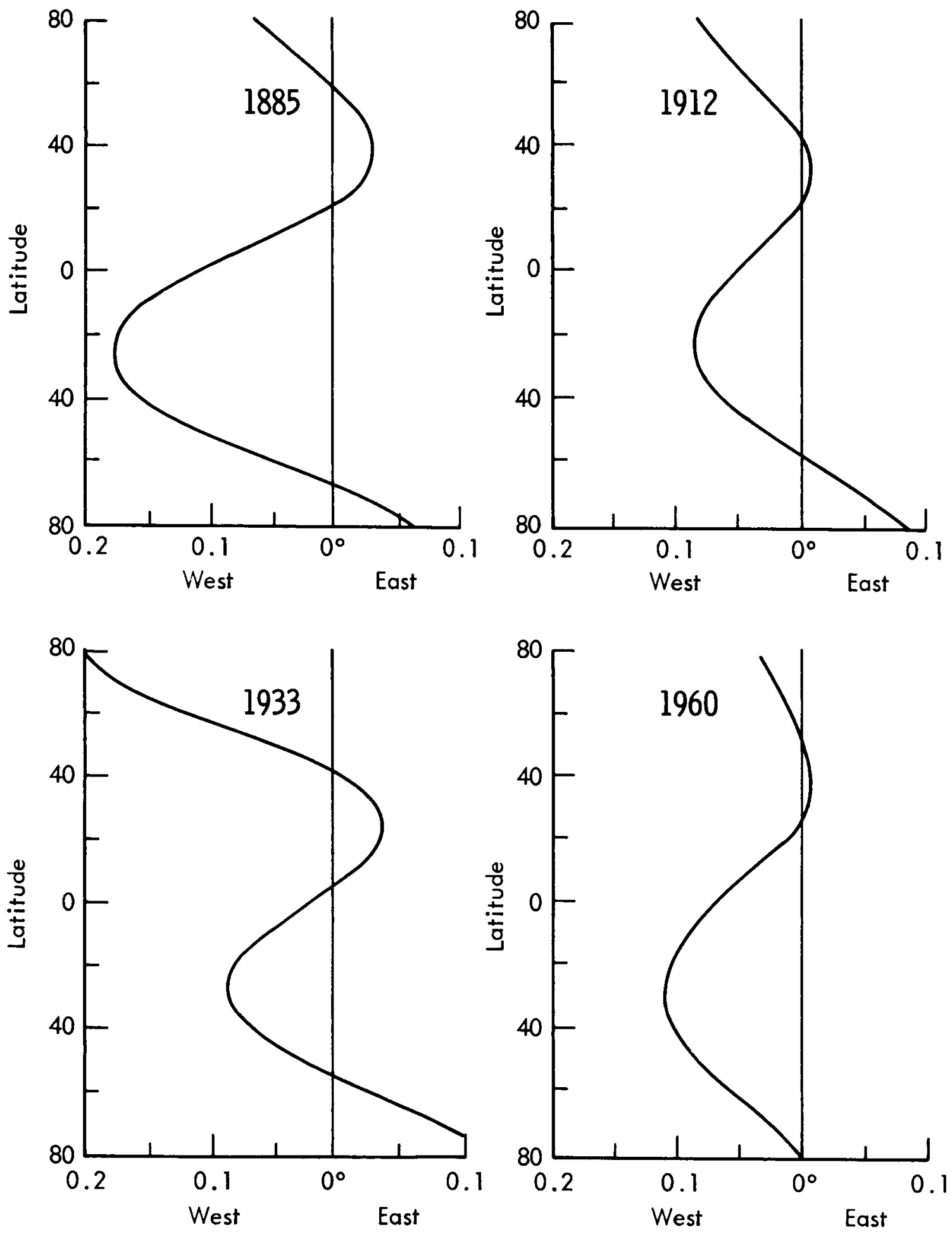

Fig. 8 -- Westward drift component of core surface velocity for epochs 1885, 1912, 1933, and 1960 . 
In view of the paucity of information suggesting the fluid nature of the earth's core, it is particularly satisfying to find that the formulation of $\underset{\sim}{\mathrm{v}}$ given in Eq. (4), which did not assume the earth to be in rotation, leads to circulations of fluid given by $\underset{\sim}{\mathbf{r}} \times \nabla \times$, which are related to the upflows and downflows in a manner consistent with the geostrophic condition. This is true even with respect to the more widespread Southern Hemisphere upflow and Northern Hemisphere downflow, as shown by the westward drift. The Coriolis force, however, is much less dominant here than in the earth's atmosphere.

The rapid growth and decay shown for these calculated circulations may suggest the presence of "core spots" reminiscent of sunspots, which also have a remarkably short time constant, and may involve considerable local interchange of magnetic and kinetic energies (Parker, 1955). How this could occur on the scale represented here by the calculated differences in flow near 1912, as compared with 1885 and 1960, is not at all clear. However, the increase in the rate of the earth's rotation near 1910, and the lessening of the rate of westward drift of the core suggested by the longitudinal motion of the eccentric dipole in the period 1885-1950 (Vestine, 1962), point toward a substantial change in angular momentum of the core.

It would be of interest to attempt to improve the estimate of the rate of westward drift near 1910, which presently rests so heavily and insecurely upon the westward drift of the second-degree harmonic in the analytical representation of the field that is inherent in the eccentric dipole mode1. As Richmond (1966) has shown, estimates made using methods such as those of Bullard, et al. (1950) also appear to rely heavily on the second-degree harmonic motion, when made for motion estimated at the earth's surface. 


\section{REFERENCES}

Alfvén, H., and C. Fälthammer, Cosmical Electrodynamics, Clarendon Press, Oxford, 1963.

Bullard, E. C., C. Freedman, H. Gellman, and J. Nixon, The westward drift of the Earth's magnetic field, Phil. Trans. Roy. Soc., 243-A, 67-92, 1950.

Cowling, T. G., Magnetohydrodynamics, Interscience, New York, 1957. Hendricks, S. J., and J. C. Cain, Magnetic field data for trappedparticle evaluations, J. Geophys. Res., 71, 346-347, 1966.

Hide, R., Free hydromagnetic oscillations of the Earth's core and the theory of the geomagnetic secular variation, Phil. Trans. Roy. Soc. London, A-259, 615-650, 1966.

Kahle, Anne B., E. H. Vestine, and R. H. Ball, Estimated surface motions of the Earth's core, accepted for publication in J. Geophys. Res, 1967.

Mauersberger, P., Betrachtungen über die zeitliche Änderung der Parameter des geomagnetischen Feldes auf Grund der vorliegenden Potentialentwicklungen, Geophysikalisches Institut Potsdam, Abhandlungen, Nr. 5, 5-58, Akademie-Ver lag-Berlin, 1952.

Nagata, T., Two main aspects of geomagnetic secular variation: westward drift and non-drifting components, Proceedings of the Benedum Earth Magnetism Symposium, Pittsburgh, 39-55, 1962.

Parker, E. N., Hydromagnetic dynamo mode1s, Astrophys. J., 122, 293-314, 1955.

Richmond, A. D., Westward Drift of the Geomagnetic Field and its Relation to Motions of the Earth's Core, The RAND Corporation, RM-5191-NASA, November 1966.

Roberts, D. H., and S. Scott, On analysis of the secular variation; (I) A hydromagnetic constraint: theory, J. Geomag. Geoelec., 17, 137-151, 1965.

Rochester, M. G., Geomagnetic westward drift and irregularities in the Earth's rotation, Phil. Trans. Roy. Soc. London, A-252, 531-555, 1960. Vestine, E. H., Influence of the Earth's core upon the rate of the Earth's rotation, Proceedings of the Benedum Earth Magnetism Symposium, Pittsburgh, 57-67, 1962. 
Vestine, E. H., and Anne B. Kahle, On the small amplitude of magnetic secular change in the Pacific area, J. Geophys. Res., 71, 527-530, 1966.

Vestine, E. H., L. Laporte, I. Lange, and W. E. Scott, The geomagnetic

field, its description and analysis, Carnegie Inst. Wash. Pub.

Yukatake, T., The westward drift of the magnetic field of the Earth,

Bu11. Earthquake Res. Inst., 40, 1-65, 1962. 